\title{
LE PHOTOLOG, UN USAGE PARTICULIER DE LA PHOTOGRAPHIE SUR INTERNET
}

\author{
Sandro Faes ${ }^{1}$
}

Avec l'émergence des technologies numériques, leur diffusion de plus en plus grande ainsi que leur facilité d'accès, le monde de la photographie et ses pratiques sont en train de vivre une énième (r)évolution. Il y a évidemment la production de l'image, facilitée et rendue relativement accessible en termes de coûts et de capacités techniques à court/ moyen terme. Cependant, ce qui va nous intéresser dans les lignes qui vont suivre est plus de l'ordre de la diffusion. Ou comment un phénomène, pur produit de l'ère numérique, à savoir le blog - défini comme étant un "site Web personnel composé essentiellement de billets d'actualité, publiés jour après jour ou au gré des humeurs et apparaissant selon un ordre anté-chronologique, les plus récents figurant en haut de

\footnotetext{
1 Assistant aux FUNDP à Namur.
}

Recherches en communication, $\mathrm{n}^{\circ} 27$ (2007). 
pages ${ }^{\prime \prime}$ - lorsqu'il fait la part belle à l'image photographique plutôt qu'à l'écriture, transforme les pratiques, en amont comme en aval.

L'objectif n'est pas de porter un regard figé sur cet usage particulier du blog nommé photolog ou photoblog 2 . Mais, à l'instar du processus sous-jacent à ce type particulier de forme d'expression aussi populaire que contemporaine, il s'agira de susciter des pistes de réflexion ou d'interprétation relatives à ce que l'image montrée de manière brute ou presque véhicule comme modes de fonctionnement "appauvris". Sans entrer dans des considérations techniques ou philosophiques, le but de ces lignes est donc d'aborder l'un des aspects de cet objet privilégié de communication visuelle, moyennement artistique selon Bourdieu, assagi par la société selon Barthes ${ }^{3}$. En effet, il serait en passe de vivre une désincarnation, de s'écarter un peu plus encore de ce qui fait sa spécificité autant que sa faiblesse artistique : "sa force d'évidence"4. Et donc de s'approcher d'une forme arbitraire du signe, entre alphabet phonétique et idéogramme, plus connoté que dénoté.

\section{Un "mélange" des genres}

L'idée a germé lors d'une escapade bâloise en mai 2005 afin de découvrir la rétrospective Jeff Wall organisée au Schauläger ${ }^{5}$. Simultanément une autre exposition se tenait au Kunstmuseum, "Covering the real, Kunst und Pressebild von Warhol bis Tillmans"6. Cette antre de la culture qu'est le musée des Beaux-arts, dans une ville où la culture muséale n'est pas un vain $\operatorname{mot}^{7}$, faisait la part belle à une certaine forme de photographie de presse, même si, c'était à travers les lunettes de l'art ${ }^{8}$. Quel lien entre les deux ?

1 L. LE MEUR, L., BEAUVAIS, Blogs pour les pros, Paris, Dunod, 2005, p.1.

2 Type de blog utilisé pour poster des photographies, accompagnées ou non d'un titre et/ou d'une légende.

3 R. BARTHES, La chambre claire, Paris, Gallimard, Le Seuil, 1980.

4 Ibid.

5 Rétrospective Jeff Wall - Photographs 1978-2004, du 30 avril 2005 au 25 septembre 2005.

6 Exposition du 1 mai 2005 au 21 août 2005.

$7 \mathrm{La}$ ville de Bâle et sa région, en Suisse, comptent proportionnellement à sa population le plus grand nombre de musées au monde. Dans les années soixante, la population vota un référendum afin d'acheter deux toiles de Picasso pour le musée de Bâle. Touché, l'artiste en offrit une troisième.

8 En comparaison nos Musées royaux des Beaux-Arts n'avaient en tout et pour tout 
L'idée, selon Bernhard Mendes Bürgi et Hartwig Fischer ${ }^{1}$, est que la photographie de presse, donc qui montre, serait devenue un centre d'intérêt artistique majeur dès les années soixante. En fait, dès que la télévision a envahi le monde de l'image. Le point de non-retour se situant aux alentours de 1963 et de la retransmission en boucle de l'assassinat de Kennedy. C'est à cet instant que se serait amorcée la véritable arrivée de la photographie dans le monde de l'art.

Toujours selon Bürgi et Fischer, les prémices de ce changement seraient à chercher du côté de Warhol qui, en 1962, s'inspirant de photos de presse pour quelques unes de ses œuvres, rendait soudainement le travail artistique de plus en plus anonyme, la signature de l'artiste devenant obsolète. Coïncidence ou pas, quelques années plus tard, le photojournalisme allait connaître les premiers signes d'essoufflement.

Depuis Warhol, 40 ans ont passé. Polke, Richter, Hamilton ou Morley ont utilisé des photos tirées de magazines ou de journaux, parfois pour les imiter en peinture. Une succession d'artistes se sont intéressés à la photographie et non plus seulement en détournant celle des autres mais en s'appropriant également la phase de production. De photographe au sens bourdieusien du terme à "artiste utilisant la photographie", l'évolution des artistes vis-à-vis de la photographie pourrait se définir de la sorte.

Dès lors, les Wall, Gurski et d'autres, ont acquis une reconnaissance en cultivant le mélange des genres, le dérèglement des sens, tant au niveau de la perception qu'à celui de la signification, le tout à travers la réalisation de clichés, de véritables tableaux, riches en connotations. Ne plus juste adhérer au réel, mais en être le narrateur, rompre avec le statisme des photographies traditionnelles, les thématiques classiques. Considérer la photo en tant que processus créatif faisant appel à plusieurs sens, en tant que vecteur complexe de significations, de sensations, faisant le lien, l'amalgame entre de nombreuses disciplines comme le rappelle Jean-François Chevrier dans un des ouvrages qu'il consacre à l'artiste canadien ${ }^{2}$.

qu'une modeste exposition de photographies de Dorothea Lange dans les lointaines années 70. Récemment, l'exposition Euro Vision, Les nouveaux européens par douze photographes de chez Magnum, a été la première vraie exposition photo ayant pris place dans les Musées Royaux.

1 Respectivement directeur et conservateur du Kunstmuseum.

2 J.-F. CHEVRIER, Jeff Wall, Paris, Hazan, 2006, p.307. 
J.W. s'est défini comme un narrateur. Aujourd'hui il se dit surtout photographe. Mais il a toujours beaucoup lu et s'est toujours intéressé à la différenciation des genres littéraires. Il regrette que la tradition picturale se soit dissociée de la littérature. À la fin des années 1980, nous avons beaucoup parlé de la photographie comme "interspace ", un espace intermédiaire, un espace de connexion entre la peinture, le cinéma et la littérature.

Et d'ajouter un peu plus loin dans le même ouvrage :

J.W. se dit aujourd'hui photographe parce que la part de la description « presque documentaire » s'est considérablement élargie dans son œuvre et qu'il rattache la description au "reportage".

La boucle serait-elle bouclée ? Est-ce aussi pour cela que la photographie acquiert peu à peu une légitimité, de marché diront certains, dans l' "Art" ? D'autant plus, si l'on note cette remarque de Bürgi \& Fischer $^{3}$ dans la préface de l'ouvrage Covering the real. Kunst und Pressebild, von Warhol bis Tillmans/Art and the Press Pictures, from Warhol to Tillmans édité lors de l'exposition éponyme :

Aujourd'hui dans la photo utilisée par les médias, il n'est plus possible de faire la distinction entre information et rituel, documentation et divertissement, regard critique et publicité. On jette juste un coup d'œil sur les photos. On ne les lit plus. On ne les critique plus, on ne cherche pas à déceler leur instrumentalisation politique et commerciale. On les consomme, tout simplement. Et la plupart d'entre nous ne pensent jamais à la réelle signification qu'elles peuvent avoir pour nous. Sarah Charlesworth ${ }^{4}$ remarqua d'ailleurs que lorsqu' il s' agit de photos, nous sommes analphabètes.

L'émergence du numérique a radicalement modifié le rapport que monsieur tout-le-monde peut avoir avec la photographie. Pour reprendre

3 B. M. BÜRGI, H. FISCHER (sous la dir. de), Covering the real. Kunst und Pressebild, von Warhol bis Tillmans/Art and the Press Pictures, from Warhol to Tillmans, Köln, DuMont Literatur und Kunst Verlag GmbH \& Co. 2005.

4 Artiste américaine active dans la tendance «conceptuelle ». Voir l'ouvrage L. Manovich, The Language of New Media, Cambridge, Massachusetts, The MIT Press, 2001. 
l'idée de Lev Manovich ${ }^{1}$ : La cyberculture, avec les nouveaux médias comme derniers vecteurs de son principe de "remixage" culturel, modifie les processus de production et de diffusion des œuvres. Les nouvelles technologies de l'information et de la communication apportent dès lors une nouvelle configuration culturelle. Or, si l'une des pratiques sociales liées à ces nouveaux médias, à savoir le blog, fait la part belle à la forme la plus classique de communication électronique qu'est le texte, il n'en demeure pas moins ouvert aux autres modes d'expression dont l'image. Image et souvent photographie qui sont considérées selon une vision très postmoderne de la chose. Omniprésence des références au passé, nouvelles harmonies issues d'amalgames d'éléments a priori hétéroclites, effacement de l'espace-temps et surtout rôle central de l'interprétation dans l'esthétique où auparavant le modernisme y plaçait l'auteur, sont autant d'indicateurs hérités de la fin des années 1970 et très présents dans la photographie actuelle.

Dans ce schéma, Internet et sa tendance à ne pas respecter ni le droit d'auteur ni la propriété intellectuelle dévaluent la rareté et en conséquence la valeur économique des œuvres. Ces critères romantiques hérités de la modernité industrielle se retrouvent donc mis à mal. Revoilà dès lors l'idée de ré-appropriation. Et les photo(b)logueurs, à l'image de Warhol et de ses mêmes dans les années soixante, de commencer à utiliser les travaux des autres dans une sorte de recombinaison et/ou de déployer une photographie proche du quotidien sous une forme que l'on pourrait paradoxalement qualifier de "journal intime collectif". En 2005, la BBC avait d'ailleurs consacré une série de reportages intitulés "Digital Citizens" où l'accent était mis sur le fait que ${ }^{2}$ :

It shows that creativity and entertainment does not have to come from Hollywood and big media, but that we all have this innate talent to tell stories and to entertain each other. Nor should this type of citizen led publishing be seen as any kind of threat. Instead, it is a chance to look «under the hood» and see what people want and like to do with their own pieces of media work, and to understand that people do it for different reasons.

1 L. MANOVICH est professeur au Département des arts visuels de l'Université de Californie. Il enseigne la pratique artistique et la théorie des nouveaux médias. Son ouvrage intitulé The Language of New Media, o.c, fait partie des plus aboutis en la matière.

2 http://news.bbc.co.uk/1/hi/technology/4728259.stm 
Most people who do it are not doing it to attract mass audience, they are doing it for themselves or family. So there is this phenomenon of niche media.

Traditional media needs to transform and evolve and open the doors so they are not lecturing us, but having conversation with us.

Mais alors que le binôme "réalité et spontanéité" prospère dans les médias, certains photologs se veulent investis d'un rôle contestataire. Ainsi, sans atteindre la réflexivité d'un Jeff Wall lorsqu'il laissait ouvertement transparaître sa révolte contre l'ostracisme et l'oppression dont les minorités en Amérique du Nord étaient victimes, certains utilisent cette nouvelle forme de comme une tribune de transmission, de contestation, de partage plus ou moins indirect d'une émotion, d'une sensation. Là où certains utilisent l'alphabet phonétique, d'autres préfèrent désormais l'image afin de s'exprimer.

Et c'est cet aspect du photolog qui est intéressant, indéfinissable et versatile. Pour se concentrer sur l'essence, disons que peut être considéré comme photolog, tout blog dont la part belle est faite presqu'exclusivement à la photographie comme moyen de communication. Seul un titre et éventuellement une légende au sens traditionnel du terme pouvant être acceptés. Ce qui est montré a dépassé, dans l'esprit de celui qui l'affiche, le diffuse, le stade que l'on pourrait appeler de « photo de vacance ». L'addition de texte n'est plus nécessaire, pour reprendre une expression éprouvée par les photologueurs : la photo se suffit à ellemême. Ou presque.

De même, dans le photolog classique la vidéo est généralement absente. Paradoxal si l'on sait combien les performances artistiques contemporaines en vogue n'hésitent pas à mélanger photo et vidéo presque sans distinction. Les photologs eux, ne semblent pas encore se confondre avec les vidéologs. Ces derniers possèdent d'ailleurs leur appellation propre : vlogs. Les phénomènes de convergence et d'hybridation tant présents dans le monde de l'art ne paraissent pas être la tendance lourde sur la toile.

\section{Que raconte le photolog?}

Ce point de divergence suffit-il à suggérer le fait que la force du photolog réside dans l'aspect narratif qu'adjoint l'auteur à l'aspect 
descriptif d'une image fixe, et ce, de manière plus ou moins explicite ? Il y a toujours plus que la dénotation. Dans le cas présent, l'auteur, le "producteur" semble jouer de manière consciente sur les attitudes narrativisantes du récepteur potentiel face à l'image. La dimension connotée est voulue, assumée et incontournable, parfois celle-ci intègre plusieurs niveaux de lecture car l'image a ici valeur de (pré)texte comme relate Philippe Marion ${ }^{1}$ :

Il faut trouver du récit, même s'il se cache ! Toute image peut être vue/lue comme un récit, toute image peut être reçue récit. Elle peut alors devenir un prétexte à des développements narratifs (des assomptions dirait Arrouye) qui la dépassent. En quelque sorte, elle fonctionnerait à la manière d'un test de Rorschach » où le sujet aurait à exprimer l'histoire qu'il perçoit dans la tache image. Bien sûr, ce genre d'attitude de réception individuelle narrativisante est aussi affaire de contexte et d'influence. Nous sommes tous exposés à ce que Roger Odin appelle des « faisceaux de déterminations » qui garantissent une cohérence et une certaine homologie dans l'interprétation collective des messages, et à fortiori des messages médiatiques. Dès lors que les faisceaux de déterminations sont forcément mobiles et évolutifs, une attitude de réception narrativisante peut donc différer - dans son intensité, sa pertinence, sa légitimité - selon les lieux et les époques.

Certains photologs vont même plus loin. Les photographies montrées se posent en véritables énigmes/jeux de l'auteur avec le spectateur. Le binôme photo-titre se révèle n'être qu'un ensemble de clefs, souvent complémentaires, comme autant d'indices permettant de comprendre le vrai message, parfois à mille lieues de l'impression première suggérée par la photographie et/ou le titre pris individuellement. Le récit est ici, encore plus que dans une photographie, explicitement lié au vrai message que veut faire passer l'auteur. Virtuel voire inexistant. Se trouverait-on dès lors, pour reprendre la terminologie de Gauthier relayée par Marion, dans la systématisation d'une extrodétermination du sens dans ce genre de photologs ? Et cette extrodétermination qui, à l'origine nécessite une idée de mouvement suggérée visuellement, est ici statique car liée à une suggestion de sens et non de

1 P. MARION, "Les images racontent-elles ?", in Recherches en Communication, numéro 8, 1997, p.133. 
mouvement. L'image peut donc être parfaitement statique, une nature morte par exemple, tout en amenant à une extrodétermination de sens de par l'idée, la sensation, l'impression, le message cachée derrière. Par exemple la disparition d'un grand-père vivant à la campagne.

Les interviews de photologueurs montrent que certains considèrent leur pratique comme égoïste, certains allant jusqu'à interdire les commentaires, leur expression devenant à sens unique. Les énigmes véhiculées par leurs posts s'avèrent être très difficilement résolues par quelqu'un ne partageant pas les mêmes faisceaux de déterminations. Le nombre de récepteurs potentiels aptes à partager le code utilisé peut parfois être très réduit, voire nul. Il n'est d'ailleurs pas rare que les curieux utilisent un moteur de recherche afin de déchiffrer les secrets des posts. Car ces messages, petits récits de vie, renferment souvent des sensations qui, si elles n'avaient été exprimées par l'image, ne l'auraient sans doute pas été par le texte traditionnel, trop explicite, trop facile d'accès.

Le photolog se fait dès lors également et surtout dans certains cas, outil d'expression, exutoire. La photographie devient prétexte à l'expression d'un état, d'un ensemble de sentiments, de sensations, souvent profondément ancré. Quelque chose d'assimilable à une Gestalt complexe. Gestalt complexe dont Mc Luhan qualifie les idéogrammes par opposition aux caractères arbitraires de l'alphabet phonétique. Citant Diringer et Innis ainsi que leurs ouvrages respectifs ${ }^{1}$, The Alphabet et Empire and communications, Mc Luhan avance la supposition selon laquelle l'ère de l'électronique marquerait les limites de la technologie de l'alphabet. Soulignant le fait qu'Innis expliquait pourquoi l'imprimé suscite le nationalisme, et non pas le tribalisme, et pourquoi il suscite des systèmes de prix et des économies de marché qui ne peuvent exister sans lui. Et Mc Luhan d'ajouter : "En un mot, Harold Innis fut la première personne à percevoir que les processus de changement dépendent étroitement des formes qu'emprunte la technologie des média. Ce sont donc des signes dénués de signification reliés à des sons qui n'en ont pas davantage qui donnent sa forme et son sens à l'homme occidental" 2 .

1 M. MC LUHAN, La galaxie Gutenberg 1, la genèse de l'homme typographique, Paris, Gallimard, 1977, p.105.

2 Ibid. 
Troublante coïncidence lorsque l'on considère que dans les photo$\log \mathrm{s}:$

- La photographie remplace l'usage de l'alphabet phonétique.

- Que chaque image est chargée de sens et a la prétention de véhiculer un tout, une notion, notamment par association d'idées, ce qui la rapproche des idéogrammes. Car chaque photo, induit dans le code privé de son auteur une relation à un moment, à une notion donnée. La signification n'est donc pas unique et peut également être changeante selon le socle culturel du récepteur, à la manière des faux amis dans l'utilisation classique du code alphabétique.

- Que cette désincarnation où la photographie apparaît comme trace de moments de vie et d'expérience personnelle rappelle Daido Moriyama et le courant japonais qui, dans les années soixanteseptante, s'opposait résolument au réalisme conventionnel, via notamment l'ouvrage Bye bye Photography ${ }^{1}$ et la revue Provoke ${ }^{2}$. Dans son rapport à l'image Moriyama cherche l'émergence de l'imperceptible, la création de l'événement en jouant avec les normes classiques d'une narration convenue. Susciter l'aléatoire et le subjectif, loin de la froideur du réalisme et dans une optique «d'être au monde».

- Cette « nouvelle » façon d'utilisation la photo s'exprime via un support nouveau, facile d'accès, de mise en œuvre, le blogging. Lieu d'échange peu contrôlable, rarement en adéquation avec les lois du marché, le photolog s'inscrit dans l'idée de contre-culture se développant sur Internet, donnant à ses praticiens un sentiment de légitimité relative.

Grâce aux nouvelles technologies, l'image photographique et l'usage qu'en font les gens est donc en train de se métamorphoser. Une sorte de réappropriation à large échelle des pratiques avant-gardistes jusque là réservées aux "artistes" ? Ou juste une mode éphémère liée à un moment numérique, les blogs ? La vérité est sans doute entre les deux.

1 D. MORIYAMA, Bye Bye Photography, Tokyo, Shashin Hyoronsha, 1972.

2 Revue photographique avant-gardiste publiée par Moriyama dans les années 1960-1970, objet de culte aujourd'hui. 
